\title{
Gender Dysphoria
}

In the upcoming fifth edition of the Diagnostic and Statistical Manual of Mental Disorders (DSM-5), people whose gender at birth is contrary to the one they identify with will be diagnosed with gender dysphoria. This diagnosis is a revision of DSM-IV's criteria for gender identity disorder and is intended to better characterize the experiences of affected children, adolescents, and adults.

\section{Respecting the Patient, Ensuring Access to Care}

DSM not only determines how mental disorders are defined and diagnosed, it also impacts how people see themselves and how we see each other. While diagnostic terms facilitate clinical care and access to insurance coverage that supports mental health, these terms can also have a stigmatizing effect.

DSM-5 aims to avoid stigma and ensure clinical care for individuals who see and feel themselves to be a different gender than their assigned gender. It replaces the diagnostic name "gender identity disorder" with "gender dysphoria," as well as makes other important clarifications in the criteria. It is important to note that gender nonconformity is not in itself a mental disorder. The critical element of gender dysphoria is the presence of clinically significant distress associated with the condition.

\section{Characteristics of the Condition}

For a person to be diagnosed with gender dysphoria, there must be a marked difference between the individual's expressed/experienced gender and the gender others would assign him or her, and it must continue for at least six months. In children, the desire to be of the other gender must be present and verbalized. This condition causes clinically significant distress or impairment in social, occupational, or other important areas of functioning.

Gender dysphoria is manifested in a variety of ways, including strong desires to be treated as the other gender or to be rid of one's sex characteristics, or a strong conviction that one has feelings and reactions typical of the other gender.

The DSM-5 diagnosis adds a post-transition specifier for people who are living full-time as the desired gender (with or without legal sanction of the gender change). This ensures treatment access for individuals who continue to undergo hormone therapy, related surgery, or psychotherapy or counseling to support their gender transition.

Gender dysphoria will have its own chapter in DSM-5 and will be separated from Sexual Dysfunctions and Paraphilic Disorders.

\section{Need for Change}

Persons experiencing gender dysphoria need a diagnostic term that protects their access to care and won't be used against them in social, occupational, or legal areas.

When it comes to access to care, many of the treatment options for this condition include counseling, cross-sex hormones, gender reassignment surgery, and social and legal transition to the desired 
gender. To get insurance coverage for the medical treatments, individuals need a diagnosis. The Sexual and Gender Identity Disorders Work Group was concerned that removing the condition as a psychiatric diagnosis-as some had suggested-would jeopardize access to care.

Part of removing stigma is about choosing the right words. Replacing "disorder" with "dysphoria" in the diagnostic label is not only more appropriate and consistent with familiar clinical sexology terminology, it also removes the connotation that the patient is "disordered."

Ultimately, the changes regarding gender dysphoria in DSM-5 respect the individuals identified by offering a diagnostic name that is more appropriate to the symptoms and behaviors they experience without jeopardizing their access to effective treatment options.

DSM is the manual used by clinicians and researchers to diagnose and classify mental disorders. The American Psychiatric Association (APA) will publish DSM-5 in 2013, culminating a 14-year revision process.

APA is a national medical specialty society whose more than 37,000 physician members specialize in the diagnosis, treatment, prevention and research of mental illnesses, including substance use disorders. Visit the APA at www.psychiatry.org . For more information, please contact APA Communications at 703-907-8640 or press@psych.org.

C 2013 American Psychiatric Association

\section{DSM-5}

Order DSM-5 and DSM-5 Collection at www.appi.org 